\title{
Stress during slaughter increases lipid metabolites and decreases oxidative stability of farmed rainbow trout (Oncorhynchus mykiss) during frozen storage
}

\author{
G. Secci ${ }^{a}$, G. Parisi ${ }^{\mathrm{a},},{ }^{*}, \mathrm{G}$. Dasilva ${ }^{\mathrm{b}}$, I. Medina ${ }^{\mathrm{b}}$ \\ ${ }^{a}$ Department of Agri-Food Production and Environmental Sciences, Section of Animal Sciences, University of Firenze, \\ Via delle Cascine 5, 50144 Firenze, Italy \\ ${ }^{\mathrm{b}}$ Instituto de Investigaciones Marinas, Consejo Superior de Investigaciones Científicas (IIM-CSIC), Eduardo Cabello 6 , \\ E-36208 Vigo, Spain
}

* Corresponding Author. Via delle Cascine, 5 - 50144 Florence (Italy). Phone: +39 0553288265; Fax: +39 055321216; email: giuliana.parisi@unifi.it

\begin{abstract}
The consequences of slaughter on the formation of lipid metabolites and oxidative stability of fish muscle during long term frozen storage $\left(-10^{\circ} \mathrm{C}\right)$ were evaluated using farmed rainbow trout killed by asphyxia in air or percussion. The level of major adenine nucleotides and their related compounds was determined in order to check the stress level during slaughter. Plasma lipid metabolites were studied through the determination of eicosanoids and docosanoids such as prostaglandins, leukotrienes, tromboxanes, isoprostanes, resolvins, hydroxides, hydroperoxides, coming from eicosapentaenoic (EPA), arachidonic (ARA), and docosahexaenoic (DHA) acids. In addition, lipid oxidative stability of fillets was monitored. Results revealed that stress during slaughter can greatly influence oxidative stress and oxidative stability of rainbow trout fillets. In fact, asphyxia, which was the most stressful, induced a higher production of some lipid mediators such as hydroperoxides and EPA-derived prostaglandins, such as 12-HpHEPE/15-HpHEPE and PGD3/PGE3. As a consequence, fillets derived from asphyxiated fish were less stable in terms of oxidative stability and showed lower shelf-life.
\end{abstract}

Key words: eicosanoids, rainbow trout, stress biomarker, lipid oxidation, TBARS

\section{Introduction}

The consequences of stress and/or muscle activity related to slaughter have been widely investigated during the last decades (Poli, Parisi, Scappini, \& Zampacavallo, 2005; van de Vis et al., 2003). In many species, slaughter stress has been shown to greatly influence the post-mortem biochemical processes, mostly by causing anaerobic glycolysis in muscle and increasing ATP degradation rate (Tejada, 2009). Moreover, stress has been proved to affect flesh quality during storage, by measuring freshness index K-value (Sigholt, Erikson, Rustad, Johansen, Nordtvedt, \& Seland, 1997), quality index (van de Vis et al., 2003), pH, color and water holding capacity (Simitzis, Tsopelakos, Charismiadou, Batzina, Deligeorgis, \& Miliou, 2014). Finally, Wendelaar Bonga (1997) reported that stress responses in fish concern complex mechanisms involving stimulation of oxygen uptake and transfer, mobilization of energy substrates, reallocation of energy away from growth and reproduction, and mainly suppressive effects on immune functions. These stress responses may in many ways resemble inflammatory processes.

Eicosanoids and other lipid metabolites derived from enzymatic and non enzymatic oxidation of polyunsaturated fatty acids (PUFAs) have been employed as markers of lipid stress conditions. It has been well documented that some of these compounds can play an important role in immune and inflammatory responses in fish (Rowley, Knight, LloydEvans, Holland, \& Vickers, 1995; Rowley et al., 2012). Particularly, 4-series leukotrienes (LT), lipoxine (LX), 12-hydroxyeicosatetraenoic acid (12-HETE), and 12-hydroxy-eicosapentaenoic acid (12-HEPE) generated through the action of lipoxygenases (LOX) were found to be produced in vitro by head kidney leukocytes extracted from rainbow trout under biological stimuli (Knight, Lloyd-Evans, Rowley, \& Barrow, 1993; Rowley et al., 2012). Moreover, cyclooxygenase (COX) products, such as 2-series prostaglandins (PG) and tromboxanes (TX), were found in leucocytes (Knight et al., 
1993) and thrombocytes (Lloyd-Evans et al., 1994) of rainbow trout underlining the role of these lipids in immunomodulation and other pro-inflammatory responses.

Recently, some authors have suggested a connection between stress conditions and the production of eicosanoids in different tissues of fish. Oxley, Jolly, Eide, Jordal, Svardal, and Olsen (2010) studied the effect of including different levels of plant sources in diet and acute stress pre-mortem on eicosanoids production and COX activity. In the same way, Olsen, Svardal, Eide, and Wargelius (2012) monitored cyclooxygenase activity in different tissues (midgut and hindgut segments, gills, liver, head kidney and white muscle) of Atlantic salmon subjected to acute stress. Results highlighted that stress had a profound effect on the intestinal eicosanoid content, inducing a temporal increase in the expression of cox2a in tissues. Moreover, Chung, Lee, and Lee (2013) assessed changes of the production of oxidized lipid products in marine fish (Oryzias latipes) after acute exposure to $\mathrm{H}_{2} \mathrm{O}_{2}$. The consequences of the increase of the enzymatic activity and the production of these lipid metabolites on final fish quality are still uncertain.

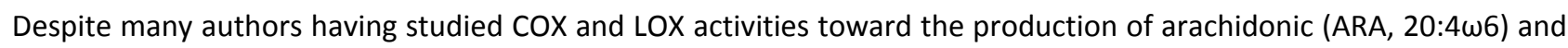
eicosapentaenoic (EPA, 22:5w3) acids-derived eicosanoids (Furne, Holen, Araujo, Lie, \& Moren, 2013; Rowley et al., 1995), in the last decade lipid products derived from oxidation of docosahexaenoic acid (DHA, 22:6w3) have been also identified. Hong, Tjonahen, Morgan, Lu, Serhan, and Rowley (2005) have reported the presence of strong antiinflammatory compounds derived from DHA, the di- and tri-hydroxy-containing bioactive products like neuroprotectin $\mathrm{D} 1(\mathrm{PD} 1)$, resolvin D5 (RvD5), resolvin D1 (RvD1) and resolvin D2 (RvD2) in rainbow trout brain cells cultures. The $14 S$ hydroxy-docosanoids acid and $17 S$-hydroxy-docosanoids acid from DHA, signatures of DHA conversion by lipoxygenases, were also identified.

This work is aimed to compare the effects of two different slaughter methods (asphyxia and percussion) on the production of lipid oxygenated mediators in plasma of farmed rainbow trout and therefore, on the shelf life of the resulting trout fillets during postmortem storage. For such scope, lipid metabolites were identified and quantified using a SPE-LC MS/MS methodology and correlated with the stress during slaughtering. As a final goal, the effect of the killing method on the oxidative stability was determined in a frozen storage experiment $\left(-10^{\circ} \mathrm{C}\right)$ by monitoring the rate of oxidation in trout fillets at different storage times. The study was completed with measurements of lipid composition in plasma and muscle of rainbow trout and the occurring degradation of nucleotides for the assessment of stress conditions during slaughter.

\section{Materials and methods}

\subsection{Preparation of fish samples and storage conditions}

Rainbow trout (Oncorhynchus mykiss) were obtain from a fish farm located in San Michele all'Adige (Trento, Trentino Alto Adige, Italy) where they have been fed with a commercial feed (42\% crude protein, $22 \%$ crude fat; Veronesi S.p.A., Verona, Italy) until $1127 \pm 258$ g. Fourteen fish were killed by percussion as control group, and other 14 were exposed to asphyxia in air until death. These two killing methods were chosen among the commonly utilized methods reviewed by EFSA (2009) because they resulted in the highest (percussion) and poorest (asphyxia) animal welfare. Immediately after death, all the fish were submitted to extraction of blood from the caudal vein and the samples were collected in heparinized syringes containing $1 \mathrm{mM}$ TRIS buffer, and 30 Units of sodium heparine, as anticoagulant, for each $\mathrm{mL}$ of final solution. Then fish were gutted and filleted, and the fillets were frozen at $-80{ }^{\circ} \mathrm{C}$. Plasma and fillets were sent to the CSIC (Consejo Superior de Investigaciones Científica) of Vigo (Spain) where samples were analyzed both for oxidative stress and oxidative stability. Firstly, plasma and muscle were characterized for fatty acid profiles (FAs). Then, ATP content of muscle and eicosanoids of plasma were evaluated as stress markers. Finally, shelf life of trout fillets was assessed by sensory analysis and the measures of secondary oxidation products of muscle, expressed as malondialdheyde (TBA-test), were monitored for 165 days maintaining samples frozen $\left(-10^{\circ} \mathrm{C}\right)$ in order to observe the oxidative stability of fillets. 
The experiment was approved according to "The regulations in Animal Experimentation" in the Department of AgriFood Production and Environmental Sciences, University of Florence and conducted by certified personnel.

\subsection{Chemical analyses}

\subsubsection{Lipid content and extraction}

Lipids were extracted from fish muscle and plasma according to the method of Bligh and Dyer (1959) and quantified gravimetrically. The extracted lipids were used for the analysis of fatty acid profiles.

\subsubsection{Fatty acid profiles of muscle and plasma}

Fatty acids were methylated according to the method of Lepage and Roy (1986) and nonadecanoic acid (C19:0) was used as an internal standard. The fatty acid methyl esters (FAMEs) were analyzed by GC according to Christie (1982).

\subsubsection{Nucleotides}

ATP and derived nucleotides were determined as proposed by Özogul, Taylor, Quantick, and Özogul (2000). Briefly, 1.5 $\mathrm{g}$ of muscle were homogenised with $10 \mathrm{~mL}$ of perchloric acid $6 \%$ (PCA, Sigma-Aldrich, St. Luis, MO, USA). After a centrifugation at $3200 \times \mathrm{g}$ at $4{ }^{\circ} \mathrm{C}$ for 10 minutes (Allegra $\mathrm{X}-12 \mathrm{R}$, Beckman Coulter Inc., Brea, CA, USA) and holding at $20^{\circ} \mathrm{C}$ for $20 \mathrm{~min}$ the samples were filtered using Filter-Lab ${ }^{\circ} 100 \mathrm{~mm}$ filter paper (Filtros Anoia, S.A., Barcelona, Spain). The extract was adjusted to $\mathrm{pH} \mathrm{6.8-7} \mathrm{using} 0.6$ and $0.1 \mathrm{M}$ potassium hydroxide (Sigma-Aldrich), filtered and finally it was brought to $25 \mathrm{~mL}$ volume with $50 \mathrm{mM}$ phosphate buffer ( $\mathrm{pH}$ 7). Before HPLC injection, $100 \mu \mathrm{L}$ of sample were filtered using a $13 \mathrm{~mm}$ GHP $0.2 \mu \mathrm{m}$ filter (Waters, Milford, MA, USA).

HPLC analyses were made using an Alliance HPLC Model 2695 (Waters, Milford, MA, USA) apparatus, equipped with a photodiode array detector model 2996 (Waters) and a multi $\lambda$ fluorescence detector (Waters). The column was a ZORBAX Eclipse XDB-C8, $4.60 \times 75 \mathrm{~mm}$, particle diameter $3.5 \mu \mathrm{m}$ (Agilent, Santa Clara, CA, USA), used at a temperature of $35^{\circ} \mathrm{C}$.

Nucleotide standards [adenosine 5'-triphosphate (ATP), adenosine $5^{\prime}$-diphosphate (ADP), adenosine 5'monophosphate (AMP), inosine $5^{\prime}$-monophosphate (IMP), inosine (Ino), hypoxanthine ( $\left.\mathrm{Hx}\right)$ ], and tetrabutylammonium bromide were purchased from Sigma-Aldrich Chemical Company (Poole, Dorset, UK). Acetonitrile was purchased from Merck KGaA (Darmstadt, Germany) and HPLC grade water was obtained from Scharlab S.L. (Sentmenat, Spain). Separation was performed in continuous gradient elution using two mobile phases. Phase A was $50 \mathrm{mM}$ phosphate buffer / $10 \mathrm{mM}$ tetrabutylammonium bromide dissolved in HPLC grade water and adjusted to pH 7 with $0.1 \mathrm{M}$ potassium hydroxide. The solution was prepared daily and filtered through a $0.2 \mu \mathrm{m} 47 \mathrm{~mm}$ GHP membrane (Waters). Phase B was acetonitrile. The injection volume was $10 \mu \mathrm{L}$ and detection was monitored at $254 \mathrm{~nm}$. The total separation time was $12 \mathrm{~min}$ with a rate flux of $1 \mathrm{~mL} / \mathrm{min}$. The results were expressed as $\mathrm{mM}$ nucleotides $/ \mathrm{g}$ muscle, and they were used to calculate K-value $=[(\mathrm{Hx}+\operatorname{lno}) /(\mathrm{Hx}+\operatorname{Ino}+\mathrm{IMP}+\mathrm{AMP}+\mathrm{ADP}+\mathrm{ATP})] * 100$ (Karube, Matsuoka, Suzuki, Watanabe, \& Toyama, 1984).

\subsubsection{Eicosanoids and docosanoids from plasma}

PUFA derivatives together with free ARA, EPA, and DHA were quantified according to the methodology previously developed by Dasilva, Pazos, Gallardo, Rodríguez, Cela, and Medina (2014) using SPE extraction prior to LC-MS/MS analysis. Briefly, plasma samples $(300 \mu \mathrm{L})$ were diluted with $30 \%$ cold methanol $(\mathrm{v} / \mathrm{v})$, to a final volume of $1.2 \mathrm{~mL}$. The internal standard 11-HETE-d8 was added to each sample. The spiked samples were incubated on ice during $10 \mathrm{~min}$ and 
then centrifuged at $3200 \times \mathrm{g}$ for $10 \mathrm{~min}$, at $4 \stackrel{\circ}{\circ}$, to remove any precipitated proteins which might cause interferences. The clear supernatant and washes of the resultant pellet with $30 \%$ methanol were collected in amber glass vials and subjected to SPE on Oasis-HLB cartridges (60 mg, 3mL; Waters, Milford, MA, USA). After that, extracts were evaporated to dryness under a fine stream of nitrogen; the residue was dissolved in $100 \mu \mathrm{L}$ ethanol and analyzed by $\mathrm{LC} / \mathrm{MS} / \mathrm{MS}$ in a Waters C18-Symmetry column, $150 \times 2.1 \mathrm{~mm}, 3.5 \mu \mathrm{m}$ (Milford, MA, USA) using a binary eluent system of water $(A)$ and methanol $(B)$, both with $0.02 \%(v / v)$ of formic acid, as mobile phases. The flow rate was set at 0.2 $\mathrm{mL} / \mathrm{min}$; the column effluent was directly introduced in the ESI without splitting, and injection volume was set to 10 $\mu \mathrm{L}$. Operating conditions of the ESI source were negative ion mode with a gas flow rate of 40 units, spray voltage of $5.5 \mathrm{kV}$, capillary temperature of $300 \stackrel{\circ}{\circ}$ and S-lens radio-frequency level of $60 \%$. The quantification of target compounds was made using the most intense, or selective, transition for each analyte and identification was helped comparing the MS/MS spectra, recorded in the range from 90 to $400 \mathrm{~m} / \mathrm{z}$ units.

\subsubsection{Sensory Analysis}

Sensory detection of rancid odors was evaluated by an expert panel formed by four trained specialists in descriptive analysis of marine off-flavors. The determination was performed in a room designed for such purpose, after the samples had been held during $10 \mathrm{~min}$ at room temperature. Approximately $10 \mathrm{~g}$ were placed in separate sterile polystyrene Petri dishes and put on a tray of ice. The panelist detected the rancidity/painty odors using a structured scale, from 8 (absolutely fresh) to 1 (putrid).

\subsubsection{TBARS}

The 2-thiobarbituric acid reactive substances (TBARS) were measured according to Vyncke (1970). The results were expressed as $\mathrm{mg}$ of malondialdehyde (MDA) equivalents/kg sample using a calibration curve determined with eight standard solutions of TEP (1,1,3,3,-Tetraethoxypropane) at concentration ranging from 0.2 to $3.1 \mu \mathrm{M}$.

\subsection{Statistical analysis}

Data were analysed using the General Linear Model procedures of the statistical analysis software SAS 9.1 (2004) for Windows. A one-way ANOVA tested the stunning method as fixed effect.

\section{Results and discussion}

\subsection{ATP degradation}

In order to test the occurrence of stress during slaughter, degradation of ATP and the formation of its corresponding catabolites were investigated in muscle. The concentration ( $\mu$ mol nucleotides / g muscle) of ADP, AMP, IMP, Ino, and $\mathrm{Hx}$ in fillets of farmed trout killed with asphyxia or percussion is shown in Table 1.

Samples taken soon after slaughter contained only low concentrations, if any, of ADP, and traces of AMP while ATP level was below the detection limit (ND) for both sample groups. These findings are in agreement with the fact that stress associated with slaughter of the fish leads to a rapid loss of ATP and initiation of the degradation sequence (Tejada, 2009).

The rapid conversion of ATP to its further catabolites was confirmed by the high amounts of IMP, and Ino. IMP was the most abundant nucleotide in both killing groups followed by Ino. Despite no significant differences being found, IMP content was lower in trout killed by asphyxia $(5.4 \mu \mathrm{mol} / \mathrm{g}$ muscle) when compared with those killed by percussion (6.1 $\mu \mathrm{mol} / \mathrm{g}$ muscle), while the opposite trend was reported for the final degradation products (Ino and Hx). These data revealed the possible influence of the killing procedures on stress, suggesting a major effect on asphyxiated fish. 
Wills, Zampacavallo, Poli, Proctor, and Henehan (2006), evaluating the effect of different slaughter methods on nucleotide contents, found an IMP concentration of 10 and $4 \mu \mathrm{mol} / \mathrm{g}$ muscle for air asphyxiated and percussion stunned rainbow trout, respectively. The IMP level obtained in this work is higher than $5.4 \mu \mathrm{mol} / \mathrm{g}$ muscle. However, that difference might be explained with the global nucleotides profile. In the present study Ino and $\mathrm{Hx}$ were also reported in abundance compared with the data of Wills et al. (2006) who had low levels for almost all nucleotides, with a very small amount of Ino and Hx. Although the degradation of ATP up to IMP is very fast, the degradation of IMP is relatively slow (Tejada, 2009) so that differences between Ino and $\mathrm{Hx}$ concentration might be attributed to a higher muscular activity due to stress during slaughter.

In order to confirm that percussive stunning method was less stressful than asphyxia, K-value (Karube et al., 1984) was calculated as index of tissue stress. Results in Table 1 show a significant higher K-value $(p<0.05)$ for the asphyxia group (29\%) than for the percussion one (18\%), demonstrating that asphyxia induced more stress in fish.

\subsection{Muscle and plasma fatty acids}

All fish used in this study showed a muscle lipid content around to $5 \%$; the total fatty acid (TFA) composition is reported in Table 2. As expected, no statistical differences were found in the lipid content between fish subjected to two killing methods, and only few statistical differences were found in the fatty acid profile of muscle. Regarding the TFA composition of plasma, no significant differences were found in its main constituents between the asphyxia and percussion groups (Table 2).

PUFA represented the majority of total fatty acids in muscle, composed primarily by $\omega 6$ and by a smaller amount of $\omega 3$. Although a certain caution is required in the comparison of the results with previous studies, since the FA composition depends considerably on diets (Baron, Svendsen, Lund, JokuSEMn, Nielsen, \& Jacobsen, 2013), the presence of vegetable sources in the feed for the farmed rainbow trout of the present trial is reflected mainly by the percentage of linoleic acid $(18: 2 \omega 6)$ that reached up $27 \%$ of TFA. Linoleic acid is the precursor for the synthesis of

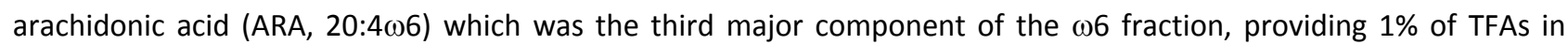
muscle.

Despite the abundance of the $\omega 6$ fraction, the PUFA composition (reported in Table 2 ) affected the $\omega 6 / \omega 3$ ratio that was 1.3, about 3 times lower than the maximum value (4.0) recommended by UK Department of Health (Committee on Medical Aspect of Food Policy, 1994).

As regards to long chain $\omega 3$ PUFAs, precursors of eicosanoids and other lipid mediators, the major contributors were

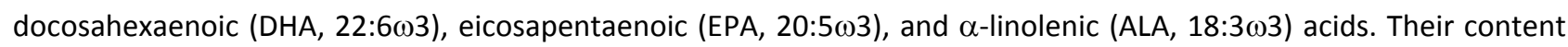
agreed with the values proposed for farmed rainbow trout fed commercial feeds (Baron et al., 2013; Blanchet, Lucas, Julien, Morin, Gingras, \& Dewailly, 2005; Haliloğlu, Bayır, Necdet Sirkecioğlu, Mevlüt Aras, \& Atamanalp, 2004) containing both fish meal and fish oil. No differences were found between asphyxia and percussion groups.

Regarding the lipid composition of plasma, the total SFAs were found to be around $25 \%$, being mainly composed by palmitic, stearic, and myristic acids. Among MUFAs, the most abundant were oleic and palmitoleic acids. Total PUFAaccounted for $58.5 \%$ of TFA of which $41 \%$ were $\omega 3$ PUFAs and $17.7 \%$ belonged to $\omega 6$ series. The most representative long chain fatty acid was DHA, followed by linoleic acid, and EPA.

\subsection{Lipid mediators}

Table 3 summarizes the plasma levels of the free fatty acids (ARA, EPA, and DHA) which are the main precursors of eicosanoids and docosanoids. The relative levels of these plasma circulating free fatty acids (FFA) showed that DHA was the preponderant free fatty acid in plasma, followed by EPA and ARA in both groups of fish. The concentration order (DHA>EPA>ARA) was in agreement with the TFA composition found in plasma. Despite no significant differences being found between methods of slaughtering in the concentration of these fatty acids, the results showed a tendency 
to lower free ARA, EPA, and DHA concentrations in asphyxiated trout than in percussion-slaughtered one. The percentage of decrease was the same for the three fatty acids (around $13 \%$ ).

The lipid mediator profile of plasma in percussion-slaughtered and asphyxiated rainbow trout presented different molecules derived from ARA, EPA, and DHA (Table 3). The oxidized products detected were: isoprostane $F_{2 \alpha}(8-$ isoPGF $F_{2 \alpha}$ ), prostaglandin $\mathrm{E}_{2}\left(\mathrm{PGE}_{2}\right), \pm 11$-hydroxy-5Z,8Z,12E,14Z-eicosatetraenoic acid (11-HETE), and leukotriene $\mathrm{B}_{4}$ $\left(\mathrm{LTB}_{4}\right)$ (from ARA); prostaglandin $\mathrm{D}_{3}$ /prostaglandin $\mathrm{E}_{3} \quad\left(\mathrm{PGD}_{3} / \mathrm{PGE}_{3}\right)$, 12(S)-hydroperoxy-5Z-,8Z,10E,14Z,17Zeicosapentaenoic acid and 15(S)-hydroperoxy-5Z,8Z,11Z,13E,17Z-eicosapentaenoic acid (12-HpEPE/15-HpEPE), \pm 5 hydroxy-6E,8Z,11Z,14Z,17Z-eicosapentaenoic acid (5-HEPE), \pm 12 - hydroxy-5Z,8Z,10E,14Z,17Z-eicosapentaenoic acid (12-HEPE), and \pm 15 -hydroxy-5Z,8Z,11Z,13E,17Z-eicosapentaenoic acid (15-HEPE) (from EPA); and \pm 4 -hydroxy-5E,7Z,10Z,13Z,16Z,19Z-docosahexaenoic acid (4-HDoHE), \pm 11 -hydroxy-4Z,7Z,9E,13Z,16Z,19Z-docosahexaenoic acid (11HDoHE), \pm 17 -hydroxy-4Z,7Z,10Z,13Z,15E,19Z-docosahexaenoic acid (17-HDoHE) (from DHA). Finally, isoprostane $F_{3 \alpha}$ (8-isoPGF $F_{3 \alpha}$ ), thromboxan $\mathrm{B}_{3}\left(\mathrm{TXB}_{3}\right.$ ) (from ARA), 17(S)-hydroperoxy-4Z,7Z,10Z,13Z,15E,19Z-docosahexaenoic acid (17HpDoHE), resolvin D1 (RvD1), and neuroprotectin D1 (PD1) (from DHA) were searched but not detected.

Considering the percussion-slaughtered as the group with less stress induced during slaughter, the results indicated that 17-HDoHE, resulting from LOX activity on DHA, appeared to be the main lipid metabolite in plasma followed by the EPA resulting product, 12-HEPE. Other products also derived from the action of LOX towards DHA, as 4-HDoHE and 11-HDoHE, were present in significant concentrations. 5-HEPE derived from EPA was also detected reaching higher concentrations than 15-HEPE. Finally, low amounts of $\mathrm{LTB}_{4}$ were also found. Hydroperoxides from EPA and DHA were below the detection limit and RvD1 and PD1 were not detected.

Concerning the COX derived products, levels of $\mathrm{PGE}_{2}$, 8-isoPGF $\mathrm{P}_{2 \alpha}$, 11-HETE, $\mathrm{PGD}_{3} / \mathrm{PGE}_{3}$, 8-isoPGF $\mathrm{P}_{3}$ and $\mathrm{TXB}_{3}$ were investigated. $\mathrm{PGE}_{2}$ was the main eicosanoid followed by 11-HETE; however, the latter was two times lower than $P G E_{2}$. The content of 8-isoPGF $2 \alpha$ was found only on the order of $10^{-6} \mathrm{ng} / \mathrm{mL}$, while no traces of $\mathrm{PGD}_{3} / \mathrm{PGE}_{3}, 8$-isoPGF $3 \alpha$, nor $\mathrm{TXB}_{3}$ were detected in the percussion group. Under these conditions, the overall lipid mediator profile of percussionslaughtered trout was the following 17-HDoHE > 12-HEPE > 4-HDoHE > 5-HEPE > 11-HDoHE > PGE $>$ 15-HEPE > 11HETE $>$ LTB $_{4}>8$-isoPGF $2 \alpha$.

Regarding the profile of asphyxiated rainbow trout, Table 3 reveals that the main difference between both methods of slaughtering was the high amount of hydroperoxides, 12-HpEPE/15-HpEPE, derived from 12 and 15 LOX activity on EPA. They were found at a concentration level three orders of magnitude higher than the other lipid metabolites. These compounds were not detected in the percussion trout. Other differences between the two methods were the lower levels of 5-HEPE, 4-HDoHE and 17-HDoHE detected in the asphyxiated trout compared with the percussionslaughtered ones. The LOX oxidation product 12-HEPE was the second main compound found in plasma of asphyxiated trout at similar concentrations to those previously mentioned in the percussion group and 11-HDoHE, 15HEPE, and $\mathrm{LTB}_{4}$ were found as well. Neither RvD1 nor PD1 were detected in the asphyxiated group.

According to the profile of the percussion group, $\mathrm{PGE}_{2}$ was the main COX product followed by $11-\mathrm{HETE}$ in the asphyxiated group; 8-isoPGF $2 \alpha$ was also detected whereas no traces of 8-iso $\mathrm{PGF}_{3 \alpha}$ nor $\mathrm{TXB}_{3}$ appeared. Interestingly, $\mathrm{PGD}_{3} / \mathrm{PGE}_{3}$ were only detected in this group and they were not found in the percussion trout. It must be also noticed that the levels of $\mathrm{PGE}_{2}$ found in the asphyxiated group were lower than those found in the percussion trout. Under this condition, the overall profile of the asphyxiated trout was 12-HEPE >>17-HDoHE > 11-HDoHE > 4-HDoHE > 5-HEPE > $\mathrm{PGE}_{2}>15-\mathrm{HEPE}>11-\mathrm{HETE}>\mathrm{LTB}_{4}>\mathrm{PGD}_{3} / \mathrm{PGE}_{3}>8$-isoPGF $2 \alpha$.

Therefore, the comparison between asphyxia and percussion groups revealed different trends for oxidized products. 12-HpEPE/15-HpEPE and $\mathrm{PGD}_{3} / \mathrm{PGE}_{3}$ were detected only in the asphyxiated trout whilst they were not found in the percussion group. Lower levels of 17-HDoHE, 5-HEPE, 4-HDoHE, 15-HEPE, PGE 2 , and 11-HETE were reported for asphyxia group than percussion, even though statistical differences $(p<0.05)$ were found only for $5-\mathrm{HEPE}, 17-\mathrm{HDOHE}$, and $\mathrm{PGE}_{2}$. 
Previous studies on fish subjected to stressing conditions occurring during confinement have investigated COX derivates products, such as prostaglandins, as biomarkers, although no clear trends for PG synthesis in response to stress could be uniquely discerned (Olsen et al., 2012; Oxley et al., 2010). According to Balvers et al. (2012) prostaglandins levels in plasma under stress stimuli were down-regulated. The results for PG found in the present study confirmed that stress condition at slaughter decreased PGE $_{2}$ levels. In contrast, Olsen et al. (2012) reported that only the isoform 2a of COX clearly responds to stress with an upregulation in the main tissues of Atlantic salmon (Salmo salar), whilst the other two (COX1 and COX2b) remain unaltered. That finding supports the results obtained for $\mathrm{PGD}_{3} / \mathrm{PGE}_{3}$, derived from EPA, which appeared only in the asphyxia group, revealing a possible increase of COX activity on EPA in response to stress. This opposite trend between $\mathrm{PGE}_{2}$ (from $A R A$ ) and $\mathrm{PGD}_{3} / \mathrm{PGE}_{3}$ (from EPA) may reflect that stressing killing methods might shift the COX activity towards EPA-derived species which are considered less inflammatory (Tocher, 2003), in order to minimize the impact of oxidative stress.

The present study also revealed that $12-\mathrm{HpEPE} / 15-\mathrm{HpEPE}$ could be considered as good markers of stress because they were highly produced under stress conditions while they were not detected in the percussion group. Hydroperoxide intermediates have been suggested to be involved in the biosynthesis of some eicosanoids in fish (Rowley, LloydEvans, Barrow, \& Serhan 1994), and the identification of HpEPE found in this work demonstrated such hypothesis. Hydroperoxy derivatives are the primary products of LOX that are easily reduced to hydroxydes by glutathione peroxidase (GPX) (Guichardant et al., 2011). This enzyme has been well characterized in fish during the past few years in order to associate its expression with stress during life. In particular, increasing stress time was associated with a down-regulation of GPX (Malandrakis, Exadactylos, Dadali, Golomazou, Klaoudatos, \& Panagiotaki, 2014). An increase of the activity of LOX, together with an inhibition of GPX activity caused by stress during slaughter, might explain the notable presence of HpEPE in the asphyxiated fish and the absence in the percussion-slaughtered ones. Moreover, the same trend in enzyme modulation seems to be confirmed by the significant decrease in other lipid mediators such as 5-HEPE and 17-HDoHE, from EPA and DHA, respectively, in the asphyxiated rainbow trout.

More recently, DHA was found to be a fairly good substrate of LOX in humans to produce various hydroxylated endproducts after reduction of the hydroperoxide intermediates by GPX. They are 4-HDoHE, 7-HDoHE, and 11-HDoHE (Lagarde, Bernoud-Hubac, Calzada, Véricel, \& Guichardant, 2013) and all of them were found in the present trial in plasma of rainbow trout. In addition to this, those derivatives of DHA have been described as precursors of a bioactive family, called resolvins and protectins, a class of compounds with active anti-inflammatory and inflammation resolving properties in mammals (Masoodi, Mir, Petasis, Serhan, \& Nicolaou, 2008). In the present study, RvD1 and PD1 were sought in plasma but they were not detected. Although in the present study neither resolvins nor protectins were detected, the presence of their precursors, 4-HDoHE, 7-HDoHE, and 11-HDoHE provided evidence of LOX activity on DHA in fish plasma. Moreover, the decreasing trend of their levels caused by the most stressful slaughter method may suggest the degradation of 4-HDoHE, 7-HDoHE, and 11-HDoHE in order to reduce stress conditions.

Therefore, results for both LOX and COX products revealed that the method of slaughtering seems to affect the oxidative response in plasma of rainbow trout in a complex way. The present findings revealed that 12- $\mathrm{HpEPE} / 15-$ $\mathrm{HpEPE}$ acids and $\mathrm{PGE}_{3} / \mathrm{PGD}_{3}$ could be considered as good stress biomarkers due to their presence in the asphyxia group but not in the percussion one. Moreover, that trend together with the reduction of PGE $_{2}$ level supported the hypothesis that slaughter stress condition shifted lipid mediator synthesis towards less inflammatory species derived from EPA. At the same time, the general decrease of secondary products derived from both EPA and DHA might be due to an inhibition of GPX activity or to a later degradation in more oxidized forms (Rowley et al., 1995). It is also important to take into consideration that increasing hydroperoxide concentrations could promote oxidative processes inducing loss of fish quality.

\subsection{Oxidative stability of fillets}

The formation of oxidation by-products measured by TBARS level in fillets from asphyxiated and percussionslaughtered trout is summarized in Fig. 1. Fillets belonging to both groups kept low values during the first 75 days of 
storage at $-10 \stackrel{\circ}{ } \mathrm{C}$. After that, fillets of the asphyxiated trout showed an increment of their TBARs values, raising from 0.27 up to $1.67 \mathrm{mg}$ MDA equivalents/ kg muscle, with the maximum level of $1.98 \mathrm{mg}$ MDA equivalents/ kg muscle at 135 days. In contrast, fillets of the percussion-slaughtered trout show a smaller increase after 105 days of storage, increasing from 0.29 to $0.52 \mathrm{mg}$ MDA equivalents/ kg muscle. As for the asphyxiated rainbow trout, the highest level of oxidation was reached after 135 days ( 0.69 mg MDA equivalents/ kg muscle).

Interestingly, difference between the two killing methods emerged after 75 days of storage, when TBARS for the asphyxiated fish started to increase. As shown in Fig. 1, asphyxiated rainbow trout started to be oxidized one month before the other group, showing a noticeably tendency to a faster increase in rancidity than the percussionslaughtered group. In addition to this, significant differences $(p<0.05)$ between killing methods were found at 135 and 165 days. In both cases, the asphyxiated trout was found to be more oxidized than the other. These results were in agreement with sensory analysis. Asphyxiated fillets showed a slight rancid off-flavor by the $105^{\text {th }}$ day of frozen storage and significant rancidity by the $135^{\text {th }}$ day. Instead, a slight rancid odor was not detected for the whole storage period in the fillets from the percussion group .

That trend seems to reveal that stressful killing method influenced oxidative stress during frozen storage, both reducing the length of induction phase and increasing the rate of lipid oxidation. Finally, since the interaction between peroxides and lipid oxidation is well known, it could be supposed that the higher level of hydroperoxide levels found in the asphyxiated rainbow trout might have negatively affected the flesh oxidative stability, as demonstrated by the highest level of lipid oxidation reached.

\section{Conclusion}

The present trial revealed that the killing method affected both the formation of lipid oxygenated metabolites and the oxidative stability of farmed rainbow trout. As a consequence of the greater enzymatic activity under stress conditions, the resulting higher concentration of hydroperoxides seemed to affect the oxidative stability of the asphyxiated rainbow trout flesh during the frozen storage, leading as result to a decrease of the shelf life of frozen trout fillets in terms of rancidity. Noticeably, 12- $\mathrm{HpEPE} / 15-\mathrm{HpEPE}$ and $\mathrm{PGE}_{3} / \mathrm{PGD}_{3}$, EPA-derived metabolites, could be considered as good markers of stress because they were highly produced under stress conditions (asphyxia) while they were not detected in the percussion group. Therefore, the present investigation indicated that slaughter method can largely affect the concentration of lipid oxygenated products and then the development of oxidation during postmortem storage. This in turn could reduce their commercial shelf-life, due to a higher susceptibility to develop rancidity.

\section{ACKNOWLEDGMENTS}

The authors gratefully acknowledge the Erasmus Placement Project for Giulia Secci's grant, the Consejo Superior de Investigaciones Científicas (CSIC) for the doctoral fellowship to Gabriel Dasilva, the ASTRO Company for the project financial supporting and the Fondazione Edmund Mach for kindly providing rainbow trout. Finally, the authors thank Mrs. González Peréz and Mrs. Comesaña for their excellent technical assistance.

\section{References}

Balvers, M. G. J., Verhoeckx, K. C. M., Meijerink, J., Bijlsma, S., Rubingh, C. M., Wortelboer, H. M., \& Witkamp, R. F. (2012). Time-dependent effect of in vivo inflammation on eicosanoid and endocannabinoid levels in plasma, liver, ileum and adipose tissue in C57BL/6 mice fed a fish-oil diet. International Immunopharmacology, 13(2), 204-214. doi:10.1016/j.intimp.2012.03.022.

Baron, C. P., Svendsen, G. H., Lund, I., JokuSEMn, A., Nielsen, H. H., \& Jacobsen, C. (2013). Organic plant ingredients in the diet of rainbow trout (Oncorhynchus mykiss): Impact on fish muscle composition and oxidative stability. European Journal of Lipid Science and Technology, 115(12), 1367-1377. doi:10.1002/ejlt.201300157. 
Blanchet, C., Lucas, M., Julien, P., Morin, R., Gingras, S., \& Dewailly, E. (2005). Fatty acid composition of wild and farmed Atlantic salmon (Salmo salar) and rainbow trout (Oncorhynchus mykiss). Lipids, 40(5), 529-531. Retrieved from http://www.ncbi.nlm.nih.gov/pubmed/16094864.

Bligh, E. G., \& Dyer, W. J. (1959). A rapid method of total lipid extraction and purification. Canadian Journal of Biochemistry and Physiology, 37(8), 911-917.

Christie, W. W. (1982). A simple procedure for rapid transmethylation of glycerolipids and cholesteryl esters. Journal of Lipid Research, 23, 1072-1075.

Chung, M. L. S., Lee, K. Y. E., \& Lee, C.-Y. J. (2013). Profiling of oxidized lipid products of marine fish under acute oxidative stress. Food and Chemical Toxicology, 53, 205-213. doi:10.1016/j.fct.2012.11.047.

Committee on Medical Aspect of Food Policy (1994). Nutritional aspect of cardiovascular disease (Report on Health and Social Subjects No 46). London: HMSO.

Dasilva, G., Pazos, M., Gallardo, J. M., Rodríguez, I., Cela, R., \& Medina, I. (2014). Lipidomic analysis of polyunsaturated fatty acids and their oxygenated metabolites in plasma by solid-phase extraction followed by LC-MS. Analytical and Bioanalytical Chemistry, 406(12), 2827-2839. doi:10.1007/s00216-014-7701-3.

EFSA (2009). Scientific opinion of the panel on animal health and welfare on a request from the European Commission. Species-specific welfare aspects of the main system of stunning and killing of farmed rainbow trout. The EFSA Journal, 1013, 1-55.

Furne, M., Holen, E., Araujo, P., Lie, K. K., \& Moren, M. (2013). Cytokine gene expression and prostaglandin production in head kidney leukocytes isolated from Atlantic cod (Gadus morhua) added different levels of arachidonic acid and eicosapentaenoic acid. Fish \& Shellfish Immunology, 34(3), 770-777. doi:10.1016/j.fsi.2012.11.044.

Guichardant, M., Chung, P., Liu, M., Calzada, C., Colas, R., Véricel, E., \& Lagarde, M. (2011). Functional lipidomics of oxidized product from polyunsaturated fatty acids. Chemistry and Physics of Lipids, 164, 544-548. doi:10.1016/j.chemphyslip.2011.05.002.

Haliloğlu, I. H., Bayır, A., Necdet Sirkecioğlu, A., Mevlüt Aras, N., \& Atamanalp, M. (2004). Comparison of fatty acid composition in some tissues of rainbow trout (Oncorhynchus mykiss) living in seawater and freshwater. Food Chemistry, 86(1), 55-59. doi:10.1016/j.foodchem.2003.08.028.

Hong, S., Tjonahen, E., Morgan, E. L., Lu, Y., Serhan, C. N., \& Rowley, A. F. (2005). Rainbow trout (Oncorhynchus mykiss) brain cells biosynthesize novel docosanoids acid-derived resolvins and protectins-mediator lipidomic analysis. Prostaglandins \& Other Lipid Mediators, 78(1-4), $107-116$. doi:10.1016/j.prostaglandins.2005.04.004.

Karube, I., Matsuoka, H., Suzuki, S., Watanabe, E., \& Toyama, K. (1984). Determination of fish freshness with an enzyme sensor system. Journal of Agricultural and Food Chemistry, 32(2), 314-319.

Knight, J., Lloyd-Evans, P., Rowley, A. F., \& Barrow, S. E. (1993). Effect of lipoxins and other eicosanoids on phagocytosis and intracellular calcium mobilisation in rainbow trout (Oncorhynchus mykiss) leukocytes. Journal of Leukocyte Biology, 54, 518-522.

Lagarde, M., Bernoud-Hubac, N., Calzada, C., Véricel, E., \& Guichardant, M. (2013). Lipidomics of essential fatty acids and oxygenated metabolites. Molecular Nutrition \& Food Research, 57(8), 1347-1358. doi:10.1002/mnfr.201200828.

Lepage, G., \& Roy, C. C. (1986). Direct transesterification of all classes of lipids in a one-step reaction. Journal of Lipid Research, 27(1), 114-120.

Lloyd-Evans, P., Barrow, S. E., Hill, D. J., Bowden, L., Rainger, G. E., Knight, J., \& Rowley, A. F. (1994). Eicosanoid generation and effects on the aggregation of thrombocytes from the rainbow trout, Oncorhynchus mykiss. Biochimica et Biophysica Acta, 1215(3), 291-299. Retrived from http:/ncbi.nlm.nih.gov/pubmed/7811714.

Malandrakis, E. E., Exadactylos, A., Dadali, O., Golomazou, E., Klaoudatos, S., \& Panagiotaki, P. (2014). Molecular cloning of four glutathione peroxidase (GPX) homologs and expression analysis during stress exposure of the marine teleost Sparus aurata. Comparative Biochemistry and Physiology. Part B, Biochemistry \& Molecular Biology, 168, 53-61. doi:10.1016/j.cbpb.2013.11.005.

Masoodi, M., Mir, A. A., Petasis, N. A., Serhan, C. N., \& Nicolaou, A. (2008). Simultaneous lipidomic analysis of three families of bioactive lipid mediators leukotrienes, resolvins, protectins and related hydroxy-fatty acids by liquid chromatography/electrospray ionisation tandem mass spectrometry. Rapid Communication Mass Spectrometry, 22(2),75-83. 
Olsen, R. E., Svardal, A., Eide, T., \& Wargelius, A. (2012). Stress and expression of cyclooxygenases (cox1, cox2a, cox2b) and intestinal eicosanoids, in Atlantic salmon, Salmo salar L. Fish Physiology and Biochemistry, 38(4), 951962. doi:10.1007/s10695-011-9581-1.

Oxley, A., Jolly, C., Eide, T., Jordal, A.-E. O., Svardal, A., \& Olsen, R. E. (2010). The combined impact of plant-derived dietary ingredients and acute stress on the intestinal arachidonic acid cascade in Atlantic salmon (Salmo salar). The British Journal of Nutrition, 103(6), 851-861. doi:10.1017/S0007114509992467.

Özogul, F., Taylor, A. M. D., Quantick, P. C., \& Özogul, Y. (2000). A rapid HPLC-determination of ATP-related compounds and its application to herring stored under modified atmosphere. International Journal of Food Science \& Technology, 35, 549-554.

Poli, B. M., Parisi, G., Scappini, F., \& Zampacavallo, G. (2005). Fish welfare and quality as affected by pre-slaughter and slaughter management. Aquaculture International, 13, 29-49.

Rowley, A. F., Lloyd-Evans, P., Barrow, S. E., \& Serhan, C. N. (1994). Lipoxin biosynthesis by trout macrophages involves the formation of epoxide intermediates. Biochemistry, 33, 856-863. Retrieved from http://www.ncbi.nlm.nih.gov/pubmed/8305432.

Rowley, A. F., Knight, J., Lloyd-Evans, P., Holland, J. W., \& Vickers, P. J. (1995). Eicosanoids and their role in immune modulation in fish - A brief overview. Fish \& Shellfish Immunology, 5(8), 549-567. doi:10.1016/S10504648(95)80041-7.

Rowley, A. F., Morgan, E. L., Taylor, G. W., Sunyer, J. O., Holland, J. W., Vogan, C. L., \& Secombes, C. J. (2012). Interaction between eicosanoids and the complement system in salmonid fish. Developmental and Comparative Immunology, 36(1), 1-9. doi:10.1016/j.dci.2011.05.007.

SAS, 2004. SAS/STAT User's Guide (Release 9.1). SAS Inst. Inc., Cary NC, USA.

Sigholt, T., Erikson, U., Rustad, T., Johansen, S., Nordtvedt, T. S., \& Seland, A. (1997). Handling stress and storage temperature affect meat quality of farmed-raised Atlantic salmon (Salmo salar). Journal of Food Science, 62(4), 898-905. doi:10.1111/j.1365-2621.1997.tb15482.x.

Simitzis, P. E., Tsopelakos, A., Charismiadou, M. A., Batzina, A., Deligeorgis, S. G., \& Miliou, H. (2014). Comparison of the effects of six stunning/killing procedures on flesh quality of sea bass (Dicentrarchus labrax, Linnaeus 1758) and evaluation of clove oil anaesthesia followed by chilling on ice/water slurry for potential implementation in aquaculture. Aquaculture Research, 45(11), 1759-1770.

Tejada, M. (2009). Fishery products: quality, safety, and authenticity. In Rehbein, H., \& Oehlenschlager, J. (Eds.), ATPderived products and K-value determination (pp. 68-88). Blackwell Publishing. United Kingdom.

Tocher, D. R. (2003). Metabolism and functions of lipids and fatty acids in teleost fish. Reviews in Fisheries Science, 11(2), 107-184.

van de Vis, H., Kestin, S., Robb, D., Oehlenschläger, J., Lambooij, B., Münkner, W., Kuhlmann, H., Kloosterboer, K., Tejada, M., Huidobro, A., Otterå, H., Roth, B., Sørensen, N.K., Akse, L., Birne, H., \& Nesvadba, P. (2003). Is humane slaughter of fish possible for industry ? Aquaculture Research, 34, 211-220.

Vyncke, W. (1970). Direct determination of the thiobarbituric acid value in trichloroacetic acid extracts of fish as a measure of oxidative rancidity. Fette Seifen Anstrichmittel, 12, 1084-1087.

Wendelaar Bonga, S. E. (1997). The stress response in fish. Physiological Reviews, 77(3), 591-625.

Wills, C. C., Zampacavallo, G., Poli, B. M., Proctor, M. R. M., \& Henehan, G. T. M. (2006). Nitrogen stunning of rainbow trout. International Journal of Food Science and Technology, 41(4), 395-398. doi:10.1111/j.13652621.2005.01082.x. 
Table 1. ATP and related catabolites mean concentration ( $\mu \mathrm{mol}$ nucleotides/g muscle) in fillets of trout slaughtered by asphyxia or percussion.

\begin{tabular}{lcccc}
\hline & Asphyxia & Percussion & Significance & rsd $^{1}$ \\
\hline ATP & N.D. & & \\
ADP & 0.259 & N.D. & \\
AMP & 0.219 & 0.397 & 0.06 \\
IMP & 5.411 & 0.098 & 0.805 & 0.03 \\
Ino & 6.109 & 0.694 & 2.38 \\
Hx & $2.021^{\mathrm{b}}$ & $1.153^{\mathrm{a}}$ & 0.036 & 0.45 \\
K-value & 0.327 & 0.200 & 0.174 & 0.12 \\
& $29.21^{\mathrm{b}}$ & $18.72^{\mathrm{a}}$ & 0.049 & 6.04
\end{tabular}

Lowercase superscript letters indicate statistically significant differences $(p<0.05)$.

${ }^{1}$ rsd: residual standard deviation.

${ }^{2}$ N.D.: not detected. 
Table 2. Total lipids of muscle and fatty acid composition (in \% of total fatty acids) of muscle and plasma of trout slaughtered by asphyxia or percussion.

\begin{tabular}{|c|c|c|c|c|c|c|c|c|}
\hline & \multicolumn{4}{|c|}{ Muscle } & \multicolumn{4}{|c|}{ Plasma } \\
\hline & Asphyxia & Percussion & Significance & $\mathrm{rsd}^{1}$ & Asphyxia & Percussion & Significance & $\mathrm{rsd}^{1}$ \\
\hline Total lipids, \% & 4.81 & 4.88 & 0.847 & 0.416 & & & & \\
\hline $14: 0$ & 2.15 & 2.08 & - & 0.097 & 1.00 & 1.05 & 0.577 & 0.111 \\
\hline $16: 0$ & 14.01 & 13.81 & 0.740 & 0.70 & 18.21 & 18.06 & 0.880 & 1.343 \\
\hline $16: 1 \omega 7$ & 2.78 & 2.72 & 0.687 & 0.185 & 1.24 & 1.20 & 0.782 & 0.193 \\
\hline 18:0 & 3.86 & 3.84 & 0.931 & 0.227 & 5.85 & 5.30 & 0.705 & 1.971 \\
\hline $18: 1 \omega 9$ & 18.34 & 18.21 & 0.854 & 0.824 & 10.18 & 10.25 & 0.891 & 0.692 \\
\hline $18: 1 \omega 7$ & $2.43^{\mathrm{a}}$ & $2.52^{\mathrm{b}}$ & 0.040 & 0.037 & 1.64 & 1.64 & 0.995 & 0.446 \\
\hline $18: 2 \omega 6$ & 27.78 & 27.24 & 0.723 & 1.755 & 11.59 & 11.56 & 0.977 & 1.241 \\
\hline $18: 3 \omega 3$ & 2.82 & 2.72 & 0.477 & 0.145 & 0.90 & 0.74 & 0.259 & 0.183 \\
\hline $20: 2 \omega 6$ & 1.20 & 1.32 & 0.091 & 0.064 & 1.79 & 1.82 & 0.951 & 0.659 \\
\hline $20: 3 \omega 6$ & 0.78 & 0.80 & 0.750 & 0.082 & 1.60 & 1.76 & 0.515 & 0.344 \\
\hline $20: 4 \omega 6$ (ARA) & 0.97 & 1.12 & 0.169 & 0.108 & $2.07^{\mathrm{a}}$ & $2.46^{\mathrm{b}}$ & 0.014 & 0.159 \\
\hline $20: 4 \omega 3$ & $0.72^{b}$ & $0.55^{\mathrm{a}}$ & 0.045 & 0.069 & 0.43 & 0.28 & 0.448 & 0.257 \\
\hline $20: 5 \omega 3$ (EPA) & 2.96 & 3.08 & 0.520 & 0.202 & 7.62 & 7.78 & 0.508 & 0.320 \\
\hline $22: 5 \omega 3$ & 1.54 & 1.45 & 0.137 & 0.057 & 2.09 & 2.01 & 0.783 & 0.390 \\
\hline $22: 6 \omega(\mathrm{DHA})$ & 14.23 & 15.07 & 0.621 & 1.907 & 29.60 & 29.90 & 0.917 & 3.959 \\
\hline ¿SFA & 20.98 & 20.66 & 0.682 & 0.896 & 26.19 & 25.53 & 0.416 & 1.068 \\
\hline ¿MUFA & 25.51 & 25.37 & 0.889 & 1.153 & 15.72 & 15.85 & 0.887 & 1.209 \\
\hline ¿PUFA & 53.50 & 53.96 & 0.652 & 1.165 & 58.01 & 58.63 & 0.679 & 1.995 \\
\hline$\omega 3$ & 22.62 & 23.29 & 0.721 & 2.116 & 40.86 & 41.01 & 0.958 & 3.649 \\
\hline$\omega 6$ & 30.88 & 30.67 & 0.887 & 1.624 & 17.15 & 17.62 & 0.729 & 1.835 \\
\hline DHA/EPA & 4.91 & 4.75 & 0.600 & 0.336 & 3.89 & 3.83 & 0.868 & 0.484 \\
\hline$\omega 6 / \omega 3$ & 1.31 & 1.38 & 0.619 & 0.162 & 0.42 & 0.44 & 0.834 & 0.081 \\
\hline DHA/ALA & 0.55 & 0.53 & 0.779 & 0.081 & 2.58 & 2.64 & 0.889 & 0.606 \\
\hline
\end{tabular}

Lowercase superscript letters indicate statistically significant differences $(p<0.05)$.

${ }^{1}$ rsd: residual standard deviation. 
Table 3. Concentration levels of FFAs (DHA, EPA, and ARA), and lipid mediators in plasma of trout slaughtered by asphyxia or percussion. Results are expressed as $\mu \mathrm{g} / \mathrm{mL}$ of plasma for FFAs and 12-HpEPE/15-HpEPE, as $\mathrm{ng} / \mathrm{mL}$ plasma for other lipid mediators.

\begin{tabular}{|c|c|c|c|c|}
\hline & Asphyxia & Percussion & Significance & $\mathrm{rsd}^{1}$ \\
\hline DHA & 1.73 & 1.96 & 0.605 & 0.594 \\
\hline EPA & 0.70 & 0.82 & 0.421 & 0.192 \\
\hline ARA & 0.25 & 0.29 & 0.713 & 0.136 \\
\hline 8-isoPGF $2 \alpha$ & $1.36 \mathrm{E}-6$ & $1.16 \mathrm{E}-6$ & 0.235 & $2.14 \mathrm{E}-7$ \\
\hline $\mathrm{PGE}_{2}$ & $2.52^{\mathrm{a}}$ & $2.84^{\mathrm{b}}$ & 0.002 & 0.091 \\
\hline 11-HETE & 0.76 & 0.86 & 0.303 & 0.123 \\
\hline LTB $_{4}$ & 0.24 & 0.23 & 0.956 & 0.247 \\
\hline $\mathrm{PGD}_{3} / \mathrm{PGE}_{3}$ & 0.13 & ND & 0.136 & 0.112 \\
\hline 12-HрEPE/15-HpEPE & 0.10 & ND & 0.134 & 0.086 \\
\hline 5-HEPE & $3.92^{\mathrm{a}}$ & $6.27^{b}$ & 0.049 & 1.350 \\
\hline 12-HEPE & 12.60 & 12.23 & 0.344 & 0.500 \\
\hline 15-HEPE & 1.25 & 1.60 & 0.092 & 0.243 \\
\hline 4-HDoHE & 4.98 & 6.58 & 0.242 & 1.740 \\
\hline 11-HDoHE & 5.48 & 5.98 & 0.115 & 0.378 \\
\hline 17-HDoHE & $8.19^{\mathrm{a}}$ & $17.58^{b}$ & 0.040 & 5.094 \\
\hline 8-isoPGF $F_{3 \alpha}$ & ND & ND & - & - \\
\hline $\mathrm{TBX}_{3}$ & ND & ND & - & - \\
\hline 17-HpDoHE & ND & ND & - & - \\
\hline RvD1 & ND & ND & - & - \\
\hline PD1 & ND & ND & - & - \\
\hline
\end{tabular}

Lowercase superscript letters indicate statistically significant differences $(p<0.05)$.

${ }^{1}$ rsd: residual standard deviation.

Fig. 1 Secondary lipid oxidation products during 165 days of frozen storage $\left(-10^{\circ} \mathrm{C}\right)$, expressed as mg MDAequivalents/ kg muscle, in fillet of trout slaughtered by asphyxia (black line) or percussion (gray line). 


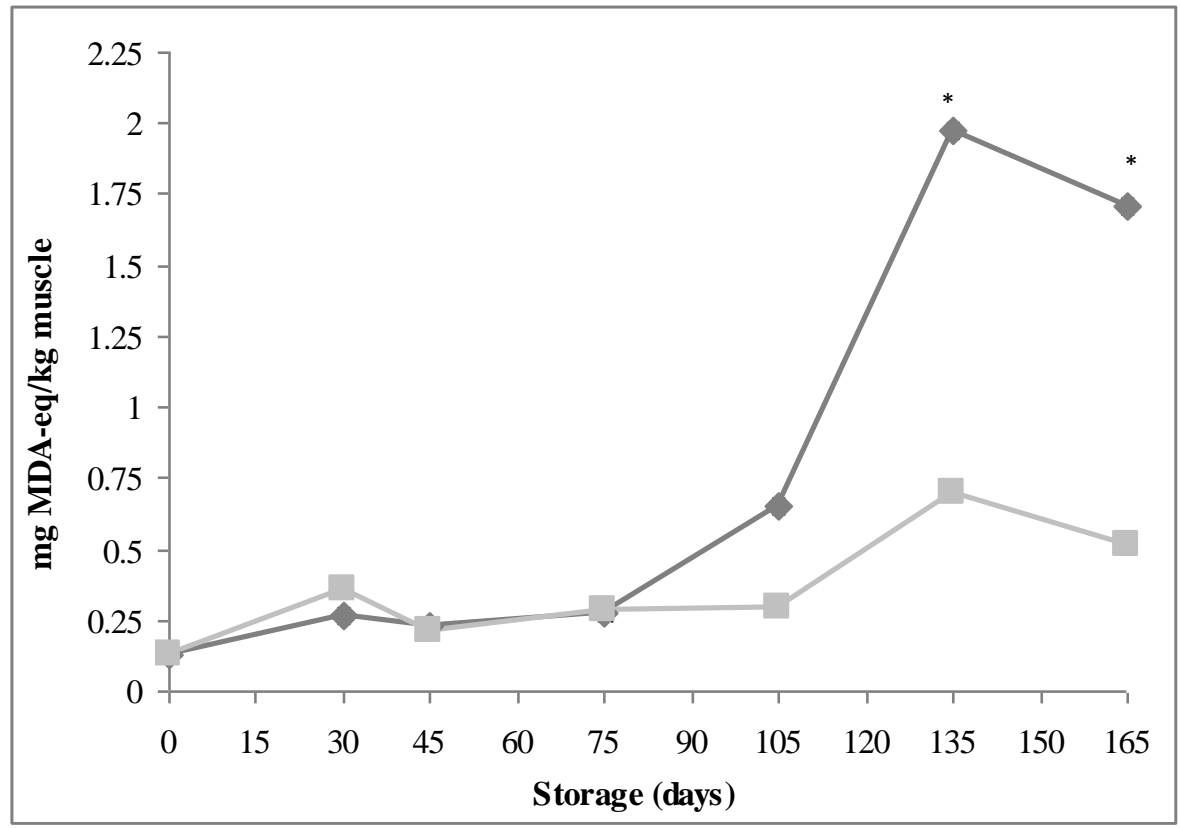

*: significant difference $(p<0.05)$ between the two groups at the specific time. 